\title{
O princípio da equivalência envolvendo cargas elétricas: a contribuição de Rohrlich para a discussão desse clássico problema
}

The equivalence principle involving electric charges: Rohrlich's contribution for the discussion on that classical problem

\author{
Edgard de Freitas Diniz Evangelista ${ }^{* 10}$ \\ ${ }^{1}$ Instituto Nacional de Pesquisas Espaciais, Divisão de Astrofísica, São José dos Campos, SP, Brasil.
}

\begin{abstract}
Recebido em 26 de abril de 2020. Revisado em 08 de outubro de 2020. Aceito em 16 de novembro de 2020.
O princípio da equivalência é um conceito fundamental da relatividade geral e traduz essencialmente a igualdade entre massa inercial e gravitacional. As idealizações a respeito do "elevador de Einstein" contendo observadores e massas de prova permitem que tal conceito seja facilmente compreensível, porém o cenário se torna mais complexo quando há cargas elétricas envolvidas em virtude de estas emitirem radiação quando aceleradas. De forma precipitada, poderíamos afirmar que uma carga em repouso em um campo gravitacional (CG) não irradia, enquanto outra dentro de um elevador acelerado em relação a um referencial inercial emite radiação, aparentemente violando o princípio da equivalência. Tal problema gerou muita discussão e mostramos aqui a análise apresentada por Fritz Rohrlich na década de 1960. O autor demonstra que o campo eletromagnético de uma carga acelerada apresenta forma radiativa apenas em alguns referenciais, não tendo tal fenômeno portando caráter absoluto. A abordagem é simples e define a curvatura do espaço-tempo como nula no domínio, não representando assim uma solução para o aparente paradoxo (pois não considera um CG real); entretanto seus resultados têm interesse didático pois servem como motivação para uma reflexão mais profunda e ajudam na compreensão de que não há incompatibilidade entre o princípio da equivalência e cargas elétricas. Essa discussão serve também como ponto de partida para trabalhos mais recentes e abrangentes sobre o problema.
\end{abstract}

Palavras-chave: Relatividade Geral, Princípio da Equivalência, Eletromagnetismo, Cargas Aceleradas.

\begin{abstract}
The equivalence principle is a fundamental concept of the general relativity and it essentially express the equality between inertial and gravitational mass. The idealizations concerning the so-called "Einstein elevator" containing observers and test masses enable us to readily understand such a concept, however the scenario becomes more complex when there are electric charges involved because they emit radiation when accelerated. Hastily, we could claim that a resting charge in a gravitational field does not radiate, while another inside an accelerated elevator relatively to an inertial frame of reference does emit radiation, seemingly violating the equivalence principle. Such a problem has stirred up many discussions and we present here the analysis put forth by Fritz Rohrlich in the 1960s. The author shows that the electromagnetic field of an accelerated charge has radiative form for some frames of reference only, thereby such a phenomenon does not have an absolute nature. The approach is simple and it defines the spacetime curvature as being null in the domain, and therefore it does not in fact represent a solution for the apparent paradox (once it does not consider a real gravitational field); however, its results have a didactic interest once they are useful as a motivation for a more profound discussion and help us to understand that there is no incompatibility between the equivalence principle and electrical charges. Also, the discussion shown here may be used as a starting point for more recent and general works on the problem.
\end{abstract}

Keywords: General Relativity, Equivalence Principle, Electromagnetism, Accelerated Charges.

\section{Introdução}

O princípio da equivalência é um dos conceitos fundamentais na formulação da teoria da relatividade geral e expressa a igualdade entre massa gravitational e inercial, a qual já havia sido demonstrada por Eötvös, entre outros [1]; veja também [2] para uma discussão interessante e abrangente sobre o assunto.

\footnotetext{
*Endereço de correspondência: edgard.freitas.diniz@gmail.com
}

Uma consequência importante da referida igualdade entre as definições de massa é que todos os corpos caem com a mesma aceleração em um CG estático e uniforme. Se considerarmos então uma caixa em queda livre, um observador no interior desta não conseguiria detectar o CG externo, já que tanto ele quanto a caixa (e quaisquer massas de prova usados no experimento) responderiam da mesma forma a tal campo [1, 3]: o observador se sentiria "flutuando", como se estivesse livre da ação de forças em um referencial inercial. 
Reciprocamente, qualquer experimento realizado no interior de uma caixa sendo uniformemente acelerada "para cima'"1 em relação a um referencial inercial produziria resultados indistinguíveis daqueles obtidos em um CG estático e uniforme. Devemos ter em mente que tais situações são válidas apenas em regiões que possam ser consideradas arbitrariamente pequenas, de forma que desvios na homogeneidade do CG (efeitos de maré) possam ser desprezados.

Embora inicialmente de fácil compreensão, o princípio da equivalência torna-se mais complicado quando há cargas elétricas em cena pelo fato de elas emitirem radiação eletromagnética quando aceleradas [4]. Por exemplo, seja a situação do parágrafo anterior: para um observador no referencial inercial haveria radiação proveniente de uma carga que estivesse em repouso em relação à caixa. Ingenuamente poderíamos pensar que se "existe" radiação, esta poderia ser também observada no próprio referencial da carga de alguma forma; o problema é que uma carga colocada, por exemplo, em repouso sobre uma mesa na superfície da Terra não irradia nesse referencial, e com isso o princípio da equivalência ficaria em cheque.

Por outro lado, um laboratório em repouso no CG detectaria a radiação proveniente de uma carga em queda livre; porém um observador em queda junto a tal corpo o perceberia imóvel em um referencial inercial, gerando um campo puramente eletrostático. Afinal, nesse caso existiria ou não radiação? Como resolver essas aparentes inconsistências?

No presente artigo abordamos uma discussão simples porém interessante de autoria de Fritz Rohrlich sobre o problema do princípio da equivalência envolvendo cargas elétricas, publicada em [5, 6] e usando resultados de 7]. O tratamento dado por Rohrlich tem como umas das características principais o fato de a radiação não ter caráter absoluto no formalismo da relatividade: é possível que um mesmo campo eletromagnético assuma configuração radiativa apenas em alguns referenciais.

O trabalho de Rohrlich é elementar em virtude de, entre outras coisas, estabelecer que a curvatura do espaço-tempo seja nula em toda a região considerada, de forma que aquilo que o autor chama de CG estático e uniforme não representa um CG real: é apenas o espaço de Minkowski submetido a uma transformação apropriada de coordenadas.

Outro ponto importante é o fato de o princípio da equivalência ser válido apenas localmente, ou seja, as caixas e laboratórios mencionados anteriormente devem ser considerados arbitrariamente pequenos; por outro lado, a radiação é um fenômeno não localizável. Assim, o campo de uma carga elétrica em queda livre em um CG real terá configuração diferente daquele gerado por uma carga idêntica em movimento inercial no espaço de Minkowski. As ponderações acima nos indicam que

\footnotetext{
1 Este termo é pouco técnico ou rigoroso porém é útil para a compreensão e será usado quando conveniente.
}

os resultados de Rohrlich não representam uma solução para o "paradoxo" e que suas alusões a CG devem ser interpretadas aqui apenas como motivação didática, servindo também para nortear o raciocínio. Nesse sentido iremos, por simplicidade e salvo alguma exceção explícita, nos referir a tal sistema não inercial como CG nas deduções a seguir.

Trabalhos mais abrangentes e recentes incluem [8], onde o autor discute os resultados de Rohrlich e acrescenta suas próprias deduções, tornando o tratamento do problema mais abrangente; interessante também é [9], o qual analisa o campo gerado por uma carga elétrica inercial do ponto de vista de observadores acelerados no contexto da gravitação semi-clássica; por fim, a série de artigos [10 14 traz estudos abrangentes sobre diversos aspectos físicos de cargas em movimento hiperbólico, com destaque para 14, onde é discutido o campo de uma carga nos espaços de Rindler e Milne.

As análises apresentadas utilizam conceitos básicos de relatividade especial e geral, além de elementos do eletromagnetismo clássico como os pontenciais de LiénardWiechert e a representação tensorial dos campos elétrico E e magnético B. Além disso, resultados e conclusões que são apenas mencionados nos artigos originais são desenvolvidos em mais detalhes aqui.

Na seção 2 mostramos o cálculo do campo gerado por uma carga em movimento retilíneo uniformemente acelerado em relação a um referencial inercial, enquanto a métrica de um CG estático e uniforme é deduzida na Seção 3 A seção 4 aborda o princípio da equivalência em si, onde são analisados três diferentes cenários, a saber: carga em repouso no CG, em queda livre e em movimento retilíneo uniformemente acelerado em relação a um observador inercial. Por fim, na Seção 5 apresentamos algumas discussões finais e conclusões.

\section{Radiação eletromagnética gerada por uma carga acelerada}

Esta seção mostra, do ponto de vista de um referencial inercial, o cálculo da radiação eletromagnética gerada por uma carga elétrica em movimento retilíneo com aceleração uniforme. Note que aqui usamos a definição relativística de aceleração uniforme, a qual é sensivelmente diferente de sua versão newtoniana. Com isso, deduzimos inicialmente a equação de movimento da carga; a partir desta e dos potenciais de Liénard-Wiechert, os campos $\mathbf{E}$ e $\mathbf{B}$ da radiação são obtidos.

\subsection{Movimento hiperbólico de um corpo}

Sejam os referenciais inerciais $S$ e $S^{\prime}$ : no caso específico em que $S^{\prime}$ se move com velocidade constante $\mathbf{v}=(0,0, v)$ na direção do eixo $z$ em relação a $S$, as coordenadas de um mesmo evento nos respectivos sistemas, isto é, $(t, x, y, z)$ em $S$ e $\left(t^{\prime}, x^{\prime}, y^{\prime}, z^{\prime}\right)$ em $S^{\prime}$, relacionam-se por 
meio das transformações de Lorentz:

$$
\begin{aligned}
& t^{\prime}=\gamma\left(t-v z / c^{2}\right), \\
& x^{\prime}=x \\
& y^{\prime}=y \\
& z^{\prime}=\gamma(z-v t),
\end{aligned}
$$

onde $\gamma=1 /\left(1-v^{2} / c^{2}\right)^{1 / 2}$ e $c$ é a velocidade da luz no vácuo. A razão de termos escolhido o eixo $z$ para o movimento da carga ficará clara na seção 2.2

Consideramos nas expressões acima que $t=t^{\prime}=0$ quando as origens de $S$ e $S^{\prime}$ coincidem e que os eixos $z$ e $z^{\prime}$ são colineares; além disso $x$ e $x^{\prime}$, assim como $y$ e $y^{\prime}$, permanecem paralelos entre si. É interessante destacar que as Equações (1)-(4) aplicam-se apenas ao caso particular do cenário em questão, enquanto uma forma mais geral de tais transformações pode ser encontrada, por exemplo, em [15.

A combinação das diferenciai: ${ }^{2}$ das Equações (1) e (4) fornece 16 ]

$$
\frac{d z^{\prime}}{d t^{\prime}}=\frac{\gamma(d z-v d t)}{\gamma\left(d t-v d z / c^{2}\right)}=\frac{(d z / d t)-v}{1-\left(v / c^{2}\right)(d z / d t)}
$$

Dessa forma, um corpo com velocidade $u=d z / d t$ medida em $S$ teria a velocidade $u^{\prime}=d z^{\prime} / d t^{\prime}$ relativa a $S^{\prime}$ dada pela Equação (5).

No caso de o objeto em questão possuir aceleração, podemos determinar como os valores desta medidos em $S$ e $S^{\prime}$ se relacionam. Com efeito, diferenciando a Equação (5), obtemos (já com a notação $u$ e $u^{\prime}$ )

$$
d u^{\prime}=\frac{1}{\gamma^{2}} \frac{d u}{\left(1-u v / c^{2}\right)^{2}}
$$

por sua vez, a combinação da Equação 6 com a diferencial da Equação (1) resulta em (veja [16] e a nota de rodapé b)

$$
\frac{d u^{\prime}}{d t^{\prime}}=\frac{1}{\gamma^{3}} \frac{1}{\left(1-u v / c^{2}\right)^{3}} \frac{d u}{d t} .
$$

Aqui consideramos o caso específico onde temos aceleração uniforme, e no contexto relativístico a definição de tal propriedade é da forma: ao longo da trajetória do corpo, todo referencial inercial $S^{\prime}$ que se colocar momentaneamente em repouso em relação a ele (ou seja, $v=u$ e $u^{\prime}=0$ ) deve medir o mesmo valor da aceleração $\alpha=d u^{\prime} / d t^{\prime}$. A aplicação de tais condições à Equação (7) leva a

$$
\alpha=\left(1-\frac{u^{2}}{c^{2}}\right)^{-3 / 2} \frac{d u}{d t}
$$

\footnotetext{
2 Tal combinação é tratada aqui como uma "divisão" das diferenciais apenas como um artifício para a simplificação dos cálculos. Rigorosamente, devemos fazer uso das regras da cadeia apropriadas.
}

A Equação (8) é facilmente resolvida para a determinação de $u(t)$; daí utilizamos $d z=u(t) d t$ para que $z(t)$, ou seja, a equação de movimento do corpo em $S$, seja obtida. Sendo $t_{0}$ o tempo inicial e fazendo $u\left(t_{0}\right)=0$, os procedimentos acima fornecem [16]

$$
z(t)=z_{0}+\left[\frac{c^{4}}{\alpha^{2}}+c^{2}\left(t-t_{0}\right)^{2}\right]^{1 / 2}-\frac{c^{2}}{\alpha}
$$

Definindo agora por simplicidade $t_{0}=0$ e $z_{0}=b$ (com $\left.b:=c^{2} / \alpha\right)$, chegamos a

$$
z_{q}(t)=\left[b^{2}+c^{2} t^{2}\right]^{1 / 2}
$$

onde o índice $q$ foi adicionado por clareza de notação.

Ao ser dividida por $b$ e após alguma álgebra, a Equação 10 assume a forma $\left[z_{q}^{2}(t) / b^{2}\right]-\left(c^{2} t^{2} / b^{2}\right)=$ 1 , daí o movimento em questão ser conhecido como hiperbólico. Perceba ainda que $u \rightarrow c$ para $t \rightarrow \infty$.

A Figura 1 apresenta o diagrama de Minkowski em termos das coordenadas $z_{q} / b$ e $c t / b$, onde a trajetória descrita pela Equação 10 é mostrada em linha sólida. Note que a região sombreada é inacessível a observadores cujo movimento obedece à Equação 10 e que a linha tracejada representando a trajetória de um raio de luz (o qual passa pela origem e viaja no sentido de $z_{q}$ positivo) define o horizonte de eventos de tais observadores.

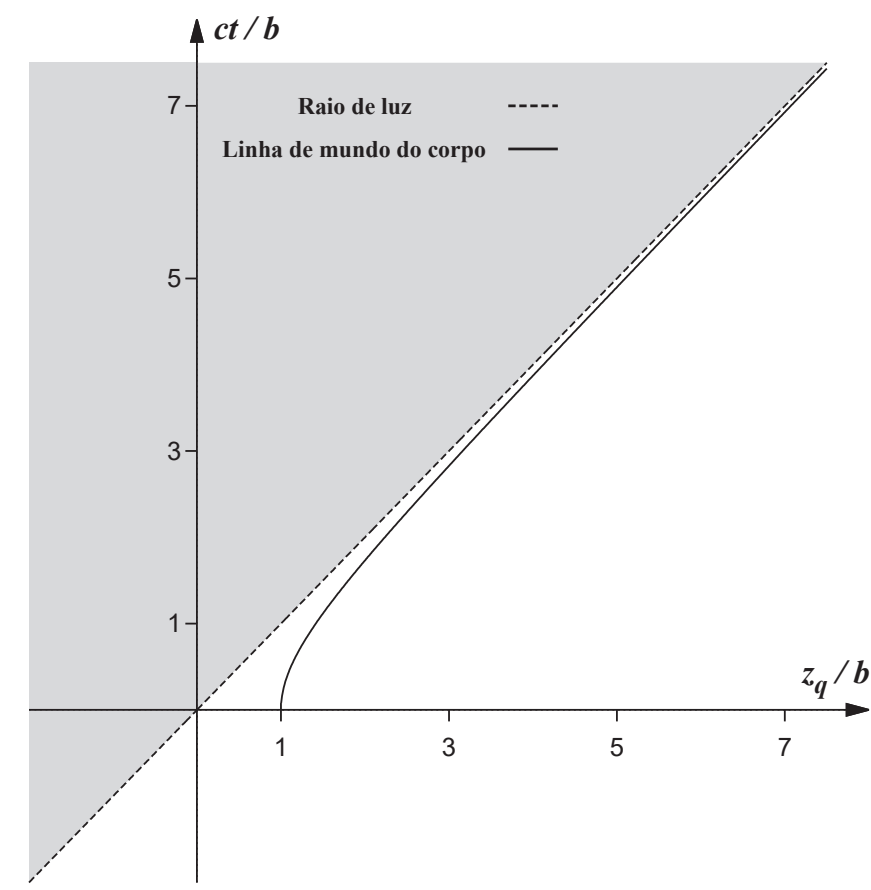

Figura 1: Diagrama de Minkowski em termos das coordenadas $z_{q} / b$ e $c t / b$. A linha sólida representa a trajetória de um corpo dada pela Equação (10); a linha tracejada indica a trajetória de um raio de luz passando pela origem e viajando no sentido de $z_{q}$ positivo. Note que tal raio delimita a região do espaço-tempo inacessível para o corpo em questão. 


\subsection{Campo gerado pela carga em termos dos potenciais}

No referencial $S$, sejam $\mathbf{r}$ e $\mathbf{r}_{q}$ as posições do observador e da carga, respectivamente. Como a radiação eletromagnética viaja à velocidade $c$, o intervalo de tempo entre sua emissão em $\mathbf{r}_{q}$ e sua detecção em $\mathbf{r}$ é $\Delta t=$ $\left|\mathbf{r}-\mathbf{r}_{q}\right| / c$; definindo agora o instante de emissão como o tempo retardado $t_{r}$, o instante de detecção $t$ é calculado por

$$
t=t_{r}+\frac{\left|\mathbf{r}-\mathbf{r}_{q}\right|}{c} .
$$

Note que na seção 2.1 o símbolo $t$ foi usado para representar, de forma genérica, a variável tempo no referencial $S$; por economia de notação, daqui por diante tal letra será usada exclusivamente para designar o tempo de detecção, conforme a Equação (11). Além disso, por conveniência serão empregadas coordenadas cilíndricas $\left(\rho=\left[x^{2}+y^{2}\right]^{1 / 2}, \phi=\tan ^{-1}[y / x]\right.$ e $\left.z=z\right)$ por todo o restante da presente seção.

A velocidade $\mathbf{v}_{q}$ da carga em $S$ é obtida pela derivada da Equação (10) em relação ao tempo e assumindo que suas outras componentes sejam nulas. Temos então

$$
\mathbf{v}_{q}\left(t_{r}\right)=\frac{c^{2} t_{r}}{\left(b^{2}+c^{2} t_{r}^{2}\right)^{1 / 2}} \hat{\mathbf{z}},
$$

a qual já aparece em termos de $t_{r}$, pois o valor de $\mathbf{v}_{q}$ a ser usado nos cálculos deve ser aquele medido no instante em que a radiação é emitida. Podemos agora calcular os potenciais escalar $V$ e vetor A gerados pela carga em movimento (os potenciais de Liénard-Wiechert) a partir de suas expressões gerais 4

$$
\begin{aligned}
V & =\frac{q}{4 \pi \varepsilon_{0}} \frac{1}{R-\boldsymbol{\beta}_{q} \cdot \mathbf{R}}, \\
\mathbf{A} & =\frac{\mu_{0} c q}{4 \pi} \frac{\boldsymbol{\beta}_{q}}{R-\boldsymbol{\beta}_{q} \cdot \mathbf{R}},
\end{aligned}
$$

onde $\boldsymbol{\beta}_{q}=\mathbf{v}_{q}\left(t_{r}\right) / c, \mathbf{R}=\mathbf{r}-\mathbf{r}_{q}, R=|\mathbf{R}|, q$ é a magnitude da carga elétrica do corpo, $\varepsilon_{0}$ é a constante de permissividade do vácuo e $\mu_{0}$ é a constante de permeabilidade do vácuo.

Como o corpo se move ao longo de $z$, a Equação 10 fornece $\mathbf{r}_{q}\left(t_{r}\right)=\left(b^{2}+c^{2} t_{r}{ }^{2}\right)^{1 / 2} \hat{\mathbf{z}}$, já em termos de $t_{r} ;$ por outro lado, temos $\mathbf{r}=\rho \hat{\boldsymbol{\rho}}+z \hat{\mathbf{z}}$. Assim $\mathbf{R}$ assume a forma

$$
\mathbf{R}=\mathbf{r}-\mathbf{r}_{q}\left(t_{r}\right)=\rho \hat{\boldsymbol{\rho}}+\left[z-\left(b^{2}+c^{2}{t_{r}}^{2}\right)^{1 / 2}\right] \hat{\mathbf{z}} .
$$

Agora $t_{r}$ pode ser explicitamente calculado substituindo a Equação 15 na Equação (11), o que resulta em uma equação de segundo grau em $t_{r}$. As operações envolvidas em tais etapas são elementares porém trabalhosas, resultando em

$$
t_{r}=\frac{t\left(z^{2}+b^{2}+\rho^{2}-c^{2} t^{2}\right) \pm(z / c) s}{2\left(z^{2}-c^{2} t^{2}\right)},
$$

onde $s=\left[\left(z^{2}+b^{2}+\rho^{2}-c^{2} t^{2}\right)^{2}-4 b^{2}\left(z^{2}-c^{2} t^{2}\right)\right]^{1 / 2}$. Na Equação (16) devemos considerar apenas o sinal negativo da solução em razão da causalidade [7] (ou seja, para assegurar que $t_{r}<0$ quando $t=0$.)

Da Equação (11) deduzimos imediatamente que $R=$ $c\left(t-t_{r}\right)$, com $t_{r}$ dado pela Equação 16 ; manipulações algébricas simples fornecem

$$
R=\frac{c t\left(z^{2}-b^{2}-\rho^{2}-c^{2} t^{2}\right)+z s}{2\left(z^{2}-c^{2} t^{2}\right)} .
$$

Por outro lado, a substituição da Equação 16 na Equação 12 permite calcular $\boldsymbol{\beta}_{q}$ de forma direta:

$$
\boldsymbol{\beta}_{q}=\frac{c t\left(z^{2}+b^{2}+\rho^{2}-c^{2} t^{2}\right)-z s}{z\left(z^{2}+b^{2}+\rho^{2}-c^{2} t^{2}\right)-c t s} \hat{\mathbf{z}} .
$$

O denominador das Equações (13) - 14 pode agora ser determinado facilmente como

$$
\begin{aligned}
R-\boldsymbol{\beta}_{q} \cdot \mathbf{R} & =c t-c t_{r}-\left|\boldsymbol{\beta}_{q}\right|\left[z-\left(b^{2}+c^{2} t_{r}{ }^{2}\right)^{1 / 2}\right] \\
& =c t-\left|\boldsymbol{\beta}_{q}\right| z
\end{aligned}
$$

com ajuda da Equação (12). As Equações (18)- 19 quando substituídas nas Equações (13)-14 fornecem finalmente

$$
\begin{aligned}
& V=\frac{q}{4 \pi \varepsilon_{0}} \frac{z\left(z^{2}+b^{2}+\rho^{2}-c^{2} t^{2}\right)-c t s}{s\left(z^{2}-c^{2} t^{2}\right)}, \\
& \mathbf{A}=\frac{\mu_{0} c q}{4 \pi} \frac{c t\left(z^{2}+b^{2}+\rho^{2}-c^{2} t^{2}\right)-z s}{s\left(z^{2}-c^{2} t^{2}\right)} \hat{\mathbf{z}} .
\end{aligned}
$$

\subsection{Cálculo de B e E}

Os campos $\mathbf{B}$ e $\mathbf{E}$ podem sem calculados em termos de $V$ e A por meio das expressões [4, 17]

$$
\mathbf{E}=-\nabla V-\frac{\partial \mathbf{A}}{\partial t} \quad \text { e } \quad \mathbf{B}=\nabla \times \mathbf{A}
$$

em coordenadas cilíndricas as componentes desses campos assumem a forma 17.

$$
\begin{aligned}
& E_{\rho}=-\frac{\partial V}{\partial \rho}-\frac{\partial A_{\rho}}{\partial t}, \quad E_{\phi}=-\frac{1}{\rho} \frac{\partial V}{\partial \phi}-\frac{\partial A_{\phi}}{\partial t}, \\
& E_{z}=-\frac{\partial V}{\partial z}-\frac{\partial A_{z}}{\partial t}, \quad B_{\rho}=\frac{1}{\rho} \frac{\partial A_{x}}{\partial \phi}-\frac{\partial A_{\phi}}{\partial x}, \\
& B_{\phi}=\frac{\partial A_{\rho}}{\partial x}-\frac{\partial A_{x}}{\partial \rho}, \quad B_{z}=\frac{1}{\rho}\left[\frac{\partial}{\partial \rho}\left(\rho A_{\phi}\right)-\frac{\partial A_{\rho}}{\partial \phi}\right] .
\end{aligned}
$$

A substituição de $\mathbf{A}=\left(A_{\rho}, A_{\phi}, A_{z}\right)$ e $V$ dados pelas Equações 2012) nas expressões acima leva a cálculos diretos porém extensos e fornece ao final (note que $A_{\rho}=$ $\left.A_{\phi}=0\right)$

$$
\begin{aligned}
& E_{\rho}=\frac{2 q b^{2}}{\pi \varepsilon_{0} s^{3}} z \rho, \\
& E_{\phi}=0,
\end{aligned}
$$




$$
\begin{aligned}
E_{z} & =\frac{q b^{2}}{\pi \varepsilon_{0} s^{3}}\left(z^{2}-c^{2} t^{2}-b^{2}-\rho^{2}\right) \\
B_{\rho} & =B_{z}=0 \\
B_{\phi} & =\frac{2 \mu_{0} c^{2} q b^{2}}{\pi s^{3}} t \rho .
\end{aligned}
$$

Podemos demonstrar que as Equações (23)-27) representam de fato um campo de radiação. Isso é feito pelo cálculo do vetor de Poynting $\mathbf{S}$ 4 correspondente a tais componentes

$$
\mathbf{S}=\frac{1}{\mu_{0}} \mathbf{E} \times \mathbf{B}=\frac{1}{\mu_{0}} B_{\phi}\left(-E_{z} \hat{\boldsymbol{\rho}}+E_{\rho} \hat{\mathbf{z}}\right),
$$

seguido pela integração de $\mathbf{S}$ no ângulo sólido $\Omega$ em torno da carga, na forma [7]

$$
\mathscr{P}=\int \mathbf{S} \cdot \hat{\mathbf{n}}\left(\frac{d t}{d t_{r}}\right) R^{2} d \Omega .
$$

O termo $d t / d t_{r}$ foi inserido na Equação 29 para que a potência total $\mathscr{P}$ da radiação emitida seja uma taxa de emissão de energia $E$ em relação a $t_{r}$, ou seja, $\mathscr{P}=d E / d t_{r}$ [7; além disso, $\hat{\mathbf{n}}=\mathbf{R} / R$. Vale notar que a Equação 29 é válida em qualquer referencial inercial e não apenas naquele em que a carga está momentaneamente em repouso no instante da emissão: tal propriedade é assegurada pelo presença do fator $d t / d t_{r}[7$.

De acordo com [7], para o cálculo da Equação (29) devemos basicamente eliminar $t$ por meio da Equação (11), reescrever os termos do integrando em função de $\cos \theta=$ $\left[z-\left(b^{2}+c^{2} t_{r}{ }^{2}\right)^{1 / 2}\right] / R$ e $\sin \theta=\rho / R$ e tomar o limite $R \rightarrow \infty$ com $t_{r}$ fixo (caso o limite seja finito e diferente de zero, configura-se a presença de radiação). Por exemplo, da Equação (11) temos

$$
\begin{aligned}
\frac{d t}{d t_{r}} & =1+\frac{1}{c} \frac{d}{d t_{r}}\left\{\rho^{2}+\left[z-\left(b^{2}+c^{2} t_{r}^{2}\right)^{1 / 2}\right]^{2}\right\}^{1 / 2} \\
& =1-\frac{c t_{r}}{\left(b^{2}+c^{2} t_{r}^{2}\right)^{1 / 2}}\left[\frac{z-\left(b^{2}+c^{2} t_{r}^{2}\right)^{1 / 2}}{R}\right] \\
& =1-\frac{c t_{r}}{\left(b^{2}+c^{2} t_{r}^{2}\right)^{1 / 2}} \cos \theta .
\end{aligned}
$$

Mais detalhes podem ser vistos em [7] e o resultado da integral dada pela Equação 29 tem a forma

$$
\mathscr{P}=\frac{2 c}{3} \frac{q^{2}}{b^{2}}=\frac{2}{3} \frac{q^{2} \alpha^{2}}{c^{3}} \text {. }
$$

A Equação (30) é justamente a fórmula de Larmor para a radiação emitida por uma carga $q$ em movimento, aplicada ao caso particular em que esta possui aceleração $\alpha$ constante [4. É útil mencionar que em [7] os autores utilizam o sistema de unidades gaussiano e $\operatorname{com} c=1$, fornecendo $\mathscr{P}=2 q^{2} \alpha^{2} / 3$.

\section{Sistema de coordenadas de um observador em repouso em um CG estático e uniforme}

Até aqui foi suficiente a utilização do formalismo da relatividade especial, uma vez que todos os cálculos têm sido feitos em relação a observadores inerciais. Por outro lado, será necessário também determinar as quantidades físicas em relação a um observador não inercial. Embora Rohrlich denomine seu referencial não inercial como um CG estático e uniforme, veremos adiante que este se trata apenas do espaço de Minkowski submetido a uma transformação de coordenadas. A métrica utilizada serve apenas como emulação de um CG em uma região suficientemente pequena, tal que a curvatura possa ser desprezada.

Considerando a convenção do somatório de Einstein e estabelecendo que todos os índices nas expressões seguintes assumem os valores 0, 1, 2 e 3, podemos escrever a métrica correspondente ao caso em questão na forma 5 .

$$
\begin{aligned}
d \sigma^{2} & =g_{\mu \nu} d x^{\mu} d x^{\nu} \\
& =-M c^{2} d \bar{t}^{2}+N d \bar{x}^{2}+P d \bar{y}^{2}+Q d \bar{z}^{2}
\end{aligned}
$$

sendo os termos $M, N, P$ e $Q$ funções apenas de $\bar{z}$; note que voltamos a usar coordenadas retangulares, definindo $\left(x^{0}, x^{1}, x^{2}, x^{3}\right)=(c \bar{t}, \bar{x}, \bar{y}, \bar{z})$, onde a barra acima das letras tem como propósito evitar confusão com o conteúdo da seção 2. Vemos ainda, a partir da Equação (31), que o tensor métrico $g_{\mu \nu}$ possui as componentes

$$
\left[g_{\mu \nu}\right]=\left(\begin{array}{cccc}
-M & 0 & 0 & 0 \\
0 & N & 0 & 0 \\
0 & 0 & P & 0 \\
0 & 0 & 0 & Q
\end{array}\right)
$$

Além de $g_{\mu \nu}$ ser função apenas de $\bar{z}$, impomos que a curvatura do espaço-tempo seja nula [5], e isso implica que a métrica deve satisfazer a equação $R_{\beta \gamma \delta}^{\alpha}=0$, onde

$$
R_{\beta \gamma \delta}^{\alpha}=\frac{\partial \Gamma_{\beta \delta}^{\alpha}}{\partial x^{\gamma}}-\frac{\partial \Gamma_{\beta \gamma}^{\alpha}}{\partial x^{\delta}}+\Gamma_{\mu \gamma}^{\alpha} \Gamma_{\beta \delta}^{\mu}-\Gamma_{\mu \delta}^{\alpha} \Gamma_{\beta \gamma}^{\mu}
$$

é o tensor de Riemann e $\Gamma_{\mu \nu}^{\alpha}$ são os símbolos de Christoffel dados por [16, 18,

$$
\Gamma_{\mu \nu}^{\alpha}=\frac{1}{2} g^{\alpha \beta}\left[\frac{\partial g_{\beta \nu}}{\partial x^{\mu}}+\frac{\partial g_{\beta \mu}}{\partial x^{\nu}}-\frac{\partial g_{\mu \nu}}{\partial x^{\beta}}\right] .
$$

Por outro lado, é usual que $R_{\beta \gamma \delta}^{\alpha}$ seja colocado na forma covariante por meio de

$$
R_{\alpha \beta \gamma \delta}=g_{\alpha \varepsilon} R_{\beta \gamma \delta}^{\varepsilon}
$$

nesse caso o tensor possuirá as simetrias $R_{\alpha \beta \gamma \delta}=$ $-R_{\beta \alpha \gamma \delta}=-R_{\alpha \beta \delta \gamma}$ e $R_{\alpha \beta \gamma \delta}=R_{\gamma \delta \alpha \beta}$, além de obedecer 
às relações de Bianchi $R_{\alpha \beta \gamma \delta}+R_{\alpha \gamma \delta \beta}+R_{\alpha \delta \beta \gamma}=0$ [16, 18.

Em princípio $R_{\alpha \beta \gamma \delta}$ teria $4^{4}=256$ componentes, porém as simetrias e as relações mencionadas no parágrafo anterior reduzem a quantidade de componentes independentes para apenas 20; de fato, no presente caso apenas seis delas são diferentes de zero, a saber ${ }^{3}$

$$
\begin{aligned}
& R_{0101}=(4 Q)^{-1} \dot{N} \dot{M}, \\
& R_{0202}=(4 Q)^{-1} \dot{P} \dot{M}, \\
& R_{1212}=-(4 Q)^{-1} \dot{N} \dot{P}, \\
& R_{0303}=2 \ddot{M}-M^{-1} \dot{M}^{2}-Q^{-1} \dot{Q} \dot{M}, \\
& R_{1313}=2 \ddot{N}-N^{-1} \dot{N}^{2}-Q^{-1} \dot{Q} \dot{N}, \\
& R_{2323}=2 \ddot{P}-P^{-1} \dot{P}^{2}-Q^{-1} \dot{Q} \dot{P},
\end{aligned}
$$

onde, para tornar as expressões mais compactas, utilizamos pontos acima das letras para indicar derivada em relação a $\bar{z}$. As Equações (36)-(41) foram obtidas pela substituição da Equação (32) nas Equação (33)-(35) e após cálculos elementares porém extensos.

Igualando as Equações (36)-(38) a zero, deduzimos que pelo menos dois dos três termos $M, N$ e $P$ devem ser constantes [5]. Por outro lado, a métrica correspondente ao limite newtoniano (CG fraco, estático e com todas as velocidades envolvidas $\ll c$ ) é dada aproximadamente por [16]

$$
d \sigma^{2}=-\left(1+\frac{2 \Phi}{c^{2}}\right) c^{2} d \bar{t}^{2}+d \bar{x}^{2}+d \bar{y}^{2}+d \bar{z}^{2},
$$

sendo $\Phi=g \bar{z}$ o potencial gravitacional [5] tal que a condição de CG fraco se traduz por $g \bar{z} / c^{2} \ll 1$. Assim, para que a Equação (31) se reduza à Equação 42 em tal limite, $M$ deve ser função de $\bar{z}$; consequentemente $N$ e $P$ são constantes e a comparação entre a Equação (31) e a Equação (42) mostra que $N=P=1$.

A bem da verdade, não temos exatamente $g_{33}=1$ no limite newtoniano (o valor é $\approx 1-2 \Phi / c^{2}$ ). Porém, como o termo temporal da métrica é multiplicado por $c^{2}$, a parte espacial só é importante ao lidarmos com o movimento da luz ou de corpos a velocidades relativísticas no CG. Consequentemente, muitos textos escrevem a métrica do limite newtoniano na forma da Equação (42): veja por exemplo a discussão na seção 9.4 de [19].

Com as deduções acima aplicadas às Equações (39)(41) igualadas a zero, ficamos com (voltando a usar a notação de Leibniz para as derivadas)

$$
2 \frac{d^{2} M}{d \bar{z}^{2}}\left(\frac{d M}{d \bar{z}}\right)^{-1}-\frac{1}{M} \frac{d M}{d \bar{z}}-\frac{1}{Q} \frac{d Q}{d \bar{z}}=0,
$$

\footnotetext{
3 Aparentemente há dois erros tipográficos no lado esquerdo da Equação (3.5) de [5]: falta uma "linha" (que indica derivada em $z$ ) no termo mais à esquerda e o sinal do termo mais à direita está trocado; em [5], a Equação (3.9) é consequência da Equação (3.5) e já não mostra tais erros.
}

a qual pode ser resolvida com o uso de $d[\ln f] / d \bar{z}=$ $f^{-1}(d f / d \bar{z})$ e fornece

$$
Q=\mathscr{Q}\left[\frac{d \sqrt{M}}{d \bar{z}}\right]^{2} .
$$

Aqui $\mathscr{Q}$ é uma constante determinada da seguinte maneira: no limite newtoniano temos $M=\left(1+2 g \bar{z} / c^{2}\right)$, daí $Q=\mathscr{Q} g^{2} /\left[c^{4}\left(1+2 g \bar{z} / c^{2}\right)\right]$; nesse caso é necessário que $\mathscr{Q}=c^{4} / g^{2}$ para que $Q \rightarrow 1$ quando $g \bar{z} / c^{2} \rightarrow 0$.

Com todas as deduções acima, a métrica procurada assume a forma

$$
d \sigma^{2}=-M c^{2} d \bar{t}^{2}+d \bar{x}^{2}+d \bar{y}^{2}+\left[\frac{c^{2}}{g} \frac{d \sqrt{M}}{d \bar{z}}\right]^{2} d \bar{z}^{2} .
$$

\subsection{Transformação de coordenadas entre a Equação (45) e a métrica de Minkowski}

Seja a métrica de Minkowski

$$
\begin{aligned}
d \mathcal{S}^{2} & =\eta_{\mu \nu} d x^{\mu} d x^{\nu} \\
& =-c^{2} d t^{2}+d x^{2}+d y^{2}+d z^{2},
\end{aligned}
$$

em que usamos as mesmas letras que na seção 2 para designar as coordenadas. Tal escolha não gera confusão e é até mesmo conveniente, como veremos na próxima seção.

Como discutido anteriormente, a métrica dada pela Equação (45) possui curvatura nula, ou seja, podemos supor que existam transformações de coordenadas por meio das quais a Equação (46) possa ser convertida na Equação (45) e vice-versa. Isso exemplifica um conhecido problema da geometria diferencial [16, a saber: descobrir o conjunto de transformações de coordenadas (ou pelo menos se existe algum) por meio do qual duas métricas conhecidas podem ser convertidas uma na outra 20 . Tal enunciado aparentemente simples é conhecido também como problema da equivalência e representa, em geral, um desafio matemático de enorme complexidade [20].

Felizmente o caso envolvendo as Equações 45 - 46 é particularmente simples e possui solução, a qual pode ser obtida de forma elementar: como um ansatz, seja a transformação $(\bar{t}, \bar{x}, \bar{y}, \bar{z}) \mapsto(t, x, y, z)$ dada pelas equações

$$
\begin{aligned}
& t=t(\bar{t}, \bar{z}) \rightarrow d t=\frac{\partial t}{\partial \bar{t}} d \bar{t}+\frac{\partial t}{\partial \bar{z}} d \bar{z}, \\
& x=\bar{x} \quad \rightarrow d x=d \bar{x}, \\
& y=\bar{y} \quad \rightarrow d y=d \bar{y} \\
& z=z(\bar{t}, \bar{z}) \rightarrow d z=\frac{\partial z}{\partial \bar{t}} d \bar{t}+\frac{\partial z}{\partial \bar{z}} d \bar{z},
\end{aligned}
$$

já com suas respectivas diferenciais; as quantidades $d t^{2}, d x^{2}, d y^{2}$ e $d z^{2}$ são então obtidas por meio das Equações (47)-(50) e substituídas na Equação (46); 
como $d^{2} \sigma=d^{2} \mathcal{S}$, as Equações $45-46$ são igualadas e chegamos a

$$
\begin{aligned}
& c^{2}\left(\frac{\partial t}{\partial \bar{t}}\right)^{2}-\left(\frac{\partial z}{\partial \bar{t}}\right)^{2}=c^{2} M \\
& \left(\frac{\partial z}{\partial \bar{z}}\right)^{2}-c^{2}\left(\frac{\partial t}{\partial \bar{z}}\right)^{2}=\left[\frac{c^{2}}{g} \frac{d \sqrt{M}}{d \bar{z}}\right]^{2} \\
& \frac{\partial z}{\partial \bar{z}} \frac{\partial z}{\partial \bar{t}}-c^{2} \frac{\partial t}{\partial \bar{z}} \frac{\partial t}{\partial \bar{t}}=0 .
\end{aligned}
$$

A Equação 51 indica que podemos fazer $\partial t / \partial \bar{t}=$ $\sqrt{M} \cosh (k \bar{t})$ e $\partial z / \partial \bar{t}=c \sqrt{M} \sinh (k \bar{t})$, onde $k$ é uma constante real a ser definida; integrando estas em $\bar{t}$ obte$\operatorname{mos} t=(\sqrt{M} / k) \sinh (k \bar{t})+t_{0}$ e $z=(c \sqrt{M} / k) \cosh (k \bar{t})+$ $z_{0}$, sendo $t_{0}$ e $z_{0}$ constantes de integração; substituindo estes resultados na Equação (52), deduzimos rapidamente que $k=g / c$. Por outro lado, $t_{0}$ e $z_{0}$ são escolhidas tal que $t=0$ e $z=c^{2} / g$ para $\bar{t}=\bar{z}=0$, fornecendo finalmente

$$
\begin{aligned}
t & =\frac{c \sqrt{M}}{g} \sinh \left(\frac{g \bar{t}}{c}\right), \\
x & =\bar{x}, \\
y & =\bar{y}, \\
z & =\frac{c^{2} \sqrt{M}}{g} \cosh \left(\frac{g \bar{t}}{c}\right) .
\end{aligned}
$$

Em [5] o autor considerou tacitamente em suas deduções que $M=1$ para $\bar{z}=0$ e tal condição foi assumida aqui também na escolha de $t_{0}$ e $z_{0}$. Isso é razoável, pois no limite newtoniano $M(\bar{z}=0)=1$. Note ainda que a Equação (53) é automaticamente satisfeita pelas Equações (54) e (57) e que a condição $z(\bar{z}=0, \bar{t}=$ $0)=c^{2} / g$ é consistente com a utilizada na dedução da Equação 10 .

\section{O princípio da equivalência envolvendo cargas elétricas}

Como discutido na seção 3, a métrica utilizada por Rohrlich, ou seja, a Equação (45), não representa um CG real. Devemos então ter em mente que os resultados desta seção valem apenas para o presente caso e não devem ser extrapolados automaticamente para cenários envolvendo CGs reais.

\subsection{Carga em repouso no $\mathrm{CG}$}

Seja uma carga elétrica em repouso em um CG como o descrito na seção 3: de que forma seria o campd ${ }^{4}$ observado no referencial da carga $S_{c g}$ ? Uma abordagem seria resolver as equações de Maxwell generalizadas para espaços-tempos curvos no presente cenário, porém essa

\footnotetext{
4 Por clareza: de agora em diante o termo "campo" sempre se referirá ao eletromagnético; o gravitacional já é escrito como CG.
}

tarefa pode se revelar extremamente complexa. Por outro lado, há uma alternativa engenhosa e relativamente simples para lidarmos com o problema, a qual será explicada a seguir.

Consideremos um referencial $S_{i}$ em queda livre no CG em questão: nele a carga mencionada no parágrafo anterior teria uma aceleração uniforme "para cima". Note que o caso aqui é o de um referencial inercial (queda livre em um CG) observando uma carga com aceleração uniforme, ou seja, é uma situação similar à descrita na seção 2 . Portanto, o campo observado em $S_{i}$ teria a forma dada pelas Equações (23)-(27). Para que o determinemos no próprio referencial da carga, basta que as Equações 23. (27) sejam transformadas tensorialmente de $S_{i}$ para $S_{c g}$ através das Equações (54)-(57), onde as coordenadas de $S_{i}$ e $S_{c g}$ são respectivamente $(c t, x, y, z)$ e $(c \bar{t}, \bar{x}, \bar{y}, \bar{z})$.

$\mathrm{Na}$ formulação tensorial do eletromagnetismo, $\mathbf{B}$ e $\mathbf{E}$ são partes do tensor de campo $F_{\mu \nu}=\partial_{\mu} A_{\nu}-\partial_{\nu} A_{\mu}$ $(\mu, \nu=0,1,2,3)$, tal que $A^{\mu}=(V / c, \mathbf{A})$ [4. Para calcularmos $F_{\mu \nu}$ no referencial $S_{i}$ fazemos inicialmente $A_{\mu}=\eta_{\mu \nu} A^{\nu}=(-V / c, \mathbf{A})$, onde $\eta_{\mu \nu}$ é o tensor métrico da Equação 46); a seguir, usando coordenadas cilíndricas dadas por $\left(x^{0}, x^{1}, x^{2}, x^{3}\right)=(c t, \rho, \phi, z) \mathrm{e}$ observando as Equações 20 - 22, deduzimos que $F_{\mu \nu}$ em $S_{i}$ tem a forma

$$
\left[F_{\mu \nu}\right]=\left(\begin{array}{cccc}
0 & -E_{\rho} / c & 0 & -E_{z} / c \\
E_{\rho} / c & 0 & 0 & -B_{\phi} \\
0 & 0 & 0 & 0 \\
E_{z} / c & B_{\phi} & 0 & 0
\end{array}\right)
$$

Perceba que, diferentemente do tensor acima, as Equações 45 - 46 estão em coordenadas retangulares. Entretanto no presente caso isso não é um problema: os movimentos tratados aqui ocorrem apenas ao longo do eixo $z$ nos respectivos referenciais, de forma que os termos das métricas envolvendo $\rho$ e $\phi$ (ou $x$ e $y$ ) não são relevantes. Além disso, $A_{\rho}=A_{\phi}=0$ e todas as grandezas eletromagnéticas em questão são independentes de $\phi$.

A transformação de $F_{\mu \nu}$ para $S_{c g}$ é na forma

$$
\bar{F}_{\alpha \beta}=\frac{\partial x^{\mu}}{\partial \bar{x}^{\alpha}} \frac{\partial x^{\nu}}{\partial \bar{x}^{\beta}} F_{\mu \nu}
$$

onde o cálculo das derivadas é feito por meio das Equações (54)-(57), porém escrevendo as Equações $\sqrt{55}-(56)$ como $\rho=\bar{\rho}$ e $\phi=\bar{\phi}$. Fazendo então $\left(\bar{x}^{0}, \bar{x}^{\perp}, \bar{x}^{2}, \bar{x}^{3}\right)=(c \bar{t}, \bar{\rho}, \bar{\phi}, \bar{z})$, temos inicialmente

$$
\begin{aligned}
& \bar{F}_{01}=-\bar{F}_{10}=-\frac{g z}{c^{3}} E_{\rho}+\frac{g t}{c} B_{\phi}, \\
& \bar{F}_{03}=-\bar{F}_{30}=-\frac{c}{2 g} \frac{d M}{d \bar{z}} E_{z}, \\
& \bar{F}_{13}=-\bar{F}_{31}=\frac{1}{2 M} \frac{d M}{d \bar{z}}\left[t E_{\rho}-z B_{\phi}\right] ;
\end{aligned}
$$

colocando $t, z, E_{\rho}, E_{z}$ e $B_{\phi}$ em termos das coordenadas $\bar{t}, \bar{\rho}, \bar{\phi}$ e $\bar{z}$ via Equações $54-57$ e após alguma álgebra, 
chegamos a

$$
\begin{aligned}
\bar{F}_{01} & =-\bar{F}_{10}=-\frac{\bar{E}_{\bar{\rho}}}{c}=-\frac{2 c^{5} q M}{\pi \varepsilon_{0} g^{3} s^{3}} \bar{\rho}, \\
\bar{F}_{03} & =-\bar{F}_{30}=-\frac{\bar{E}_{\bar{z}}}{c} \\
& =-\frac{q c^{9}}{2 \pi \varepsilon_{0} g^{5} s^{3}} \frac{d M}{d \bar{z}}\left[M-1-\frac{\bar{\rho}^{2} g^{2}}{c^{4}}\right], \\
\bar{F}_{13} & =-\bar{F}_{31}=-\bar{B}_{\bar{\phi}}=0,
\end{aligned}
$$

onde

$$
s=\frac{c^{4}}{g^{2}}\left[\left(M+1+\frac{g^{2} \bar{\rho}^{2}}{c^{4}}\right)^{2}-4 M\right]^{1 / 2} .
$$

As únicas componentes de $\bar{F}_{\alpha \beta}$ diferentes de zero são $\bar{F}_{01}, \bar{F}_{10}, \bar{F}_{03}$ e $\bar{F}_{30}$ e estas são independentes de $\bar{t}$. Notamos então que o campo descrito por $\bar{F}_{\alpha \beta}$ é de natureza puramente eletrostática, o que nos leva ao primeiro resultado a ser destacado:

Resultado 1 Uma carga elétrica em repouso no " $C G$ " em questão não emite radiação quando observada em seu próprio referencial.

Por fim, é instrutivo investigar rapidamente a forma de $\mathbf{E}$ em $S_{c g}$ : seja seu módulo calculado como $\bar{E}^{2} / c^{2}=\bar{F}^{i 0} \bar{F}_{0 i}=g^{i r} g^{0 s} \bar{F}_{r s} \bar{F}_{0 i}=g^{11} g^{00} \bar{F}_{10} \bar{F}_{01}+$ $g^{33} g^{00} \bar{F}_{30} \bar{F}_{03}$. A partir da Equação 45 , vemos que $g^{00}=-M^{-1}, g^{11}=1$ e $g^{33}=\left[\left(c^{2} / g\right)(d \sqrt{M} / d \bar{z})\right]^{-2}$; com isso e as Equações (63)-(64), ficamos com

$$
\bar{E}=\frac{q g^{2}}{\pi \varepsilon_{0} c^{4}\left[\left(M+1+g^{2} \bar{\rho}^{2} / c^{4}\right)^{2}-4 M\right]} .
$$

Na aproximação de campo fraco, ou seja, usando $M=$ $1+2 g z / c^{2}, \bar{E}$ tem a forma

$$
\bar{E}=\frac{q g^{2}}{\pi \varepsilon_{0}\left[4 g^{2}\left(\bar{z}^{2}+\bar{\rho}^{2}\right)+4 g^{3} \bar{\rho}^{2} \bar{z} / c^{2}+g^{4} \bar{\rho}^{4} / c^{4}\right]} ;
$$

como estamos desprezando a curvatura, podemos calcular a distância radial $R$ a partir do centro da carga da mesma forma que no espaço euclidiano, ou seja, $R^{2}=\bar{z}^{2}+\bar{\rho}^{2}$. Com isso e desconsiderando os termos proporcionais a $c^{-2}$ e $c^{-4}$ no denominador da Equação 68 , temos o campo aproximadamente coulombiano $\bar{E} \approx$ $q /\left(4 \pi \varepsilon_{0} R^{2}\right)$.

Concluímos que as Equações (67)- 68 representam campos coulombianos "deformados" pela presença do CG. A Figura 2 mostra o perfil de $\mathbf{E}$ de uma carga no referencial dado pela métrica da Equação 45 para o caso $M=1+2 g z / c^{2}$ e usando coordenadas $g \rho / c^{2}$ e $g z / c^{2}$ (painel superior); para comparação, é mostrado também o campo puramente coulombiano de uma carga em um referencial inercial (painel inferior). Em ambos, para melhor visualização, os campos são normalizados
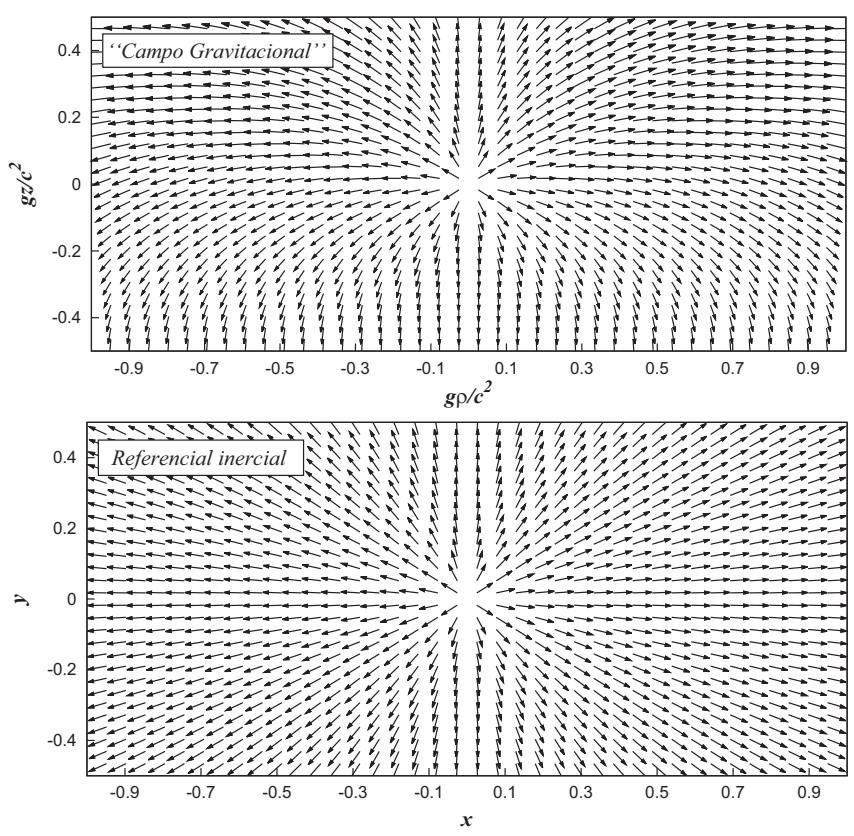

Figura 2: Em cima: perfil de $\mathbf{E}$ para uma carga em repouso no referencial dado pela Equação (45) para o caso $M=1+$ $2 g z / c^{2}$; embaixo: campo coulombiano da mesma carga em um referencial inercial. Em ambos os casos $\mathbf{E}$ é normalizado pelos respectivos módulos.

por seus respectivos módulos; além disso em ambas as situações as cargas estão em repouso na origem dos respectivos sistemas de coordenadas.

Para um tratamento sofisticado com respeito a formas de campos na presença de gravidade, o leitor pode consultar 21].

\subsection{Carga e observador com aceleração uniforme "para cima" em relação a um referencial inercial}

Seja agora o caso de uma carga elétrica com aceleração uniforme $\alpha$, cujo movimento em relação ao eixo $z$ de um referencial inercial $S_{i}$ seja descrito pela Equação (10). Inicialmente, seguindo a conhecida expressão da dilatação temporal $d t=\gamma d \tau$, onde $d t$ é o intervalo de tempo medido em $S_{i}$ e $d \tau$ o intervalo de tempo próprio no referencial da carga [16], podemos calcular $\tau(t)$ na forma

$$
\int_{\tau_{0}}^{\tau} d \tau^{*}=\int_{t_{0}}^{t} \frac{1}{\gamma} d t^{*}=\int_{t_{0}}^{t}\left[1-\left(\frac{v_{q}\left(t^{*}\right)}{c}\right)^{2}\right]^{1 / 2} d t^{*}
$$

Os asteriscos indicam variáveis de integração, enquanto $v_{q}(t)$ é dada pela Equação 12 ; note que aqui já não fazemos alusão a $t_{r}$ e sim a $t$. Substituindo a expressão de $v_{q}(t)$ na Equação 69 , obtemos a integral

$$
\tau-\tau_{0}=\int_{t_{0}}^{t}\left[1-\frac{\left(c t^{*}\right)^{2}}{b^{2}+\left(c t^{*}\right)^{2}}\right]^{1 / 2} d t^{*}
$$


a qual pode ser encontrada em tabelas ou resolvida por meio de ferramentas online como a disponível em https://www.integral-calculator.com. Assim, considerando $\tau_{0}=t_{0}=0$ e lembrando que $b=c^{2} / \alpha$, a integral fornece

$$
\tau=\frac{c}{\alpha} \sinh ^{-1}\left(\frac{\alpha t}{c}\right)
$$

A inversão da Equação 71 resulta em $t=$ $(c / \alpha) \sinh (\alpha \tau / c) ; \quad$ substituindo este resultado na Equação (10), obtemos a coordenada $z_{q}$ da carga em $S_{i}$ e ficamos com

$$
\begin{aligned}
& t=\frac{c}{\alpha} \sinh \left(\frac{\alpha \tau}{c}\right), \\
& z_{q}=\frac{c^{2}}{\alpha} \cosh \left(\frac{\alpha \tau}{c}\right) .
\end{aligned}
$$

Note que, a não ser pelo termo $\sqrt{M}$ e pelas letras diferentes para designar a aceleração, as Equações (54) e (57) têm a mesma forma das expressões acima: comparando ambos os conjuntos de relações, vemos que tanto $\tau$ quanto $\bar{t}$ são os tempos nos referenciais da carga, enquanto o lado esquerdo de cada conjunto representa coordenadas de referenciais inerciais.

Podemos portanto aplicar aqui a mesma análise usada no cenário envolvendo a carga em repouso no CG: o campo observado em $S_{i}$ tem a forma dada pelas Equações (23)-(27), sendo portanto radiativo; por outro lado, no referencial acelerado da carga tal campo teria a forma eletrostática dada pela Equação 67 com $M=1$. Somos então levados ao segundo resultado importante:

Resultado 2 Uma carga elétrica acelerada em relação a um referencial inercial não emite radiação do ponto de vista de observadores coacelerados com a carga.

A ausência de um fator como o $\sqrt{M}$ nas Equações (72)-73 é justificada pelo fato de a métrica dada pela Equação 45 representar um CG "real", no qual existe um potencial dependente das coordenadas (apenas $\bar{z}$ em nosso caso), e $\sqrt{M}$ incorpora tal característica. Por outro lado, no presente cenário não há CG envolvido: existe apenas uma carga acelerada uniformemente em relação a um observador inercial. Tal detalhe não compromete a equivalência entre as duas situações: com efeito, se na seção 4.1 considerarmos o observador no plano $\bar{z}=0$, ambos os casos tornam-se formalmente idênticos pois $M(\bar{z}=0)=1$. A similaridade entre as Equações (54)-(57) e as Equações $\sqrt{72}-(73)$ decorre do fato de estas representarem essencialmente o mesmo sistema físico, ou seja, a Equação 45 é a métrica de um referencial de Minkowski acelerado. Com efeito, se na seção 4.1 considerarmos o observador no plano $\bar{z}=0$, ambos os conjuntos de transformações tornamse formalmente idênticos pois $M(\bar{z}=0)=1$.

\subsection{Carga em queda livre do CG}

É interessante analisar ainda o caso de uma carga em queda livre no CG: é fácil deduzir que, do ponto de vista de um referencial $S_{i}$ em queda junto desta, o campo observado seria eletrostático e coulombiano. Por outro lado, em relação a um referencial $S_{c g}$ em repouso no CG, a carga descreveria um movimento retilíneo acelerado. Somos inicialmente tentados a deduzir então que o campo observado em $S_{c g}$ teria a forma das Equações 23-27). Porém não é esse o caso por conta de um fato importante: $S_{c g}$ não é inercial.

Em [5] o autor indica um modo de se calcular os campos em $S_{c g}$, o qual consiste em escrevermos $F_{\mu \nu}$ nas coordenadas de $S_{i}$ e a seguir transformá-lo para $S_{c g}$ por meio da Equação (59) e das Equações (54)-(57). Usando coordenadas cilíndricas $\left(x^{0}, x^{1}, x^{2}, x^{3}\right)=(c t, \rho, \phi, z)$, as componentes não nulas de $F_{\mu \nu}$ em $S_{i}$ têm a seguinte forma

$$
\begin{aligned}
& F_{01}=-F_{10}=-\frac{E_{\rho}}{c}=-\frac{E}{c} \frac{\rho}{R}, \\
& F_{03}=-F_{30}=-\frac{E_{z}}{c}=-\frac{E}{c} \frac{z}{R},
\end{aligned}
$$

onde $E=q /\left(4 \pi \varepsilon_{0} R^{2}\right)$ e $R^{2}=\rho^{2}+z^{2}$, com a carga na origem. Aplicando as transformações para as coordenadas $(c \bar{t}, \bar{\rho}, \bar{\phi}, \bar{z})$ em $S_{c g}$, temos as componentes não nulas

$$
\begin{aligned}
& \bar{F}_{01}=-\bar{F}_{10}=-\frac{\sqrt{M}}{c} \frac{\rho E}{R} \cosh \left(\frac{g \bar{t}}{c}\right), \\
& \bar{F}_{03}=-\bar{F}_{30}=-\frac{c}{2 g} \frac{d M}{d \bar{z}} \frac{z E}{R}, \\
& \bar{F}_{13}=-\bar{F}_{31}=\frac{c}{2 g \sqrt{M}} \frac{\rho E}{R} \frac{d M}{d \bar{z}} \sinh \left(\frac{g \bar{t}}{c}\right),
\end{aligned}
$$

com $z, \rho$ e $R$ já escritos em termos das novas coordenadas.

Os resultados acima não são mostrados em [5], porém o autor afirma que estes são similares aos encontrados em 22. De fato, transformando as Equações $76-(78)$ para coordenadas retangulares e considerando a métrica de Møller [5, 23] onde $M=\left(1+\Phi / c^{2}\right)^{2}$ (a mesma usada em [22]), obtemos expressões semelhantes às Equações (31) de [22]. As diferenças devem-se às convenções de sinal diferentes das usadas aqui e ao fato de em [22] o movimento ser na direção $x$.

Nas referências supracitadas existe discussão a respeito da natureza das Equações (76]- 778 : em [22] há a conclusão de que elas não representam um campo de radiação, enquanto [5] afirma o contrário. O argumento apresentado em 22] considera uma carga nos momentos $t$ e $-t$, nos quais ela tem mesma posição e velocidades de mesmo módulo porém sentidos opostos; o cálculo de $T_{\mu \nu}$ permite deduzir que em ambos os instantes as distribuições de densidade de energia e momento do campo são as mesmas; por outro lado, o vetor de Poynting tem mesma magnitude mas difere em sinal e, 
dada a simetria entre ambas as situações e notando que a energia mecânica da partícula é a mesma, o autor conclui então que entre $t$ e $-t$ não há energia sendo retirada do sistema via radiação.

Já em [5] afirma-se que as Equações $(76)-(78)$ representam radiação com a condição de que consideremos apenas os campos retardados, ou seja, a situação em que $t>t_{r}$ (ou $R>0$, sendo os campos avançados caracterizados por $R<0$ ). O raciocínio é como segue: as situações em $t$ e $-t$ de 22 são interpretadas respectivamente como configurações simétricas de campos retardados e avançados, cujas contribuições para a radiação se cancelam exatamente; sem o campo avançado, a simetria é quebrada e portanto existe radiação total emitida.

Segundo [5, 7], esse desacordo quanto à existência de radiação deve-se ao fato de a condição $R>0$ não ser automaticamente satisfeita pelos potenciais estudados aqui, devendo ser assegurada explicitamente. Com efeito, note que a condição de causalidade $t>t_{r}$ imposta em meio às deduções da seção 2.2 já se perde na Equação (17): vemos que $R$ em princípio pode ser negativo, visto que não há restrições a priori quanto aos valores de $z, \rho$ e $t[7]$.

\section{Discussões finais e conclusões}

Nas seções anteriores não mencionamos o fenômeno da reação à radiação, e além disso foi tacitamente considerado que corpos neutros e carregados caem com a mesma aceleração em um CG. Em princípio isso pode soar inconsistente, já que a radiação emitida deve exercer uma força de reação sobre a carga, fazendo com que esta caia com aceleração menor do que a de um objeto neutro, sendo a energia conservada no processo: a aceleração diminui pois uma parcela da energia mecânica e do momento são transportadas pela radiação. Mas veja que tal força de reação para uma carga movendo-se no eixo $z$ é calculada como [4, 17] (também conhecida como força de Abraham-Lorentz)

$$
F_{\text {rad }}=\frac{\mu_{0} q^{2}}{6 \pi c} \frac{d^{3} z}{d t^{3}}=\frac{q^{2}}{6 \pi \varepsilon_{0} c^{3}} \frac{d \alpha}{d t},
$$

a qual é zero em movimentos retilíneos uniformemente acelerados já que $\alpha$ é constante.

Deduzimos que, embora haja radiação, $F_{\text {rad }}=0 \mathrm{em}$ nosso caso e corpos carregados e neutros desenvolvem a mesma aceleração em queda livre. Isso torna tudo confuso pois parece que a energia não é mais conservada. Qual a fonte da energia transportada pelas ondas? Alguns autores chegaram mesmo a deduzir que cargas em aceleração uniforme não irradiam em virtude de não haver força de reação, com destaque para Richard Feynman 24.

Em sua abordagem Rohrlich considerou que a energia da radiação vem do próprio campo ao redor da carga, onde fica confinada na forma da chamada "energia de
Schott" 25]. Assim, quando uma carga acelera uniformemente, a potência da radiação é exatamente a taxa de variação negativa da energia de Schott [7, 25]. Tais conceitos são ainda bastante obscuros, com várias interpretações e controvérsias: veja por exemplo [4, 7], [17, 25, 26], entre vários outros estudos.

Outra questão que deve ser respondida é a respeito da situação em que a carga está acelerada em relação a um referencial inercial. Nesse caso, por que observadores coacelerados não detectam a radiação? Para onde ela "vai"? Mais uma vez o assunto é complexo e vamos adotar o argumento apresentado em [8, 27, colocado aqui de forma muito resumida: conforme discutido na seção 2.1 (veja Figura 1), referenciais acelerados possuem horizontes de eventos e portanto há partes do espaçotempo que lhes são inacessíveis; acontece que a radiação de cargas coaceleradas "cai" nessas regiões tornandose indetectáveis para tais observadores, os quais perceberiam apenas um campo coulombiano "deformado" e sem natureza radiativa. Argumento semelhante pode ser aplicado ao cenário da carga em queda livre.

Concluindo, apresentamos aqui a abordagem de F. Rohrlich para o problema do princípio da equivalência envolvendo cargas elétricas. Analisamos os cenários com carga em repouso no CG, em queda livre e acelerada uniformemente em relação a um referencial inercial, nos quais deduzimos de forma geral que observadores coacelerados com a carga não detectam radiação.

Como sumário, a Figura 3 esquematiza as situações deduzidas no presente artigo. Os painéis da esquerda mostram os casos em que a radiação é observada, e de cima para baixo, temos: observador em repouso no CG e carga em queda livre; observador em queda livre e carga em repouso no CG; e observador acelerado em relação a uma carga inercial. As imagens à direita (e de cima para baixo) representam os cenários em que não há radiação em relação ao observador: este e a carga em repouso no CG; ambos em queda livre no CG; ambos acelerados em relação a um referencial inercial.

Os resultados apresentados aqui devem ser considerados apenas como uma contribuição para o entendimento da aparente incompatibilidade entre o princípio da equivalência e cargas elétricas, e isso decorre principalmente do fato de o "CG" considerado por Rohrlich não ser real; com efeito, dado que a curvatura é definida como sendo nula, tal sistema não inercial é apenas a métrica de Minkowski submetida a uma transformação de coordenadas. Sendo assim, os sistemas descritos aqui como "CG estático e uniforme" e "acelerado para cima" correspondem à mesma situação física, e um tratamento que pretenda de fato verificar de forma mais geral o princípio da equivalência com cargas elétricas deve incluir um CG real. No entanto, o trabalho abordado no presente artigo é interessante do ponto de vista histórico, já que serviu de base para diversos outros trabalhos; além disso, ele serve como motivação e introdução para tratamentos mais recentes e abrangentes sobre o problema. 


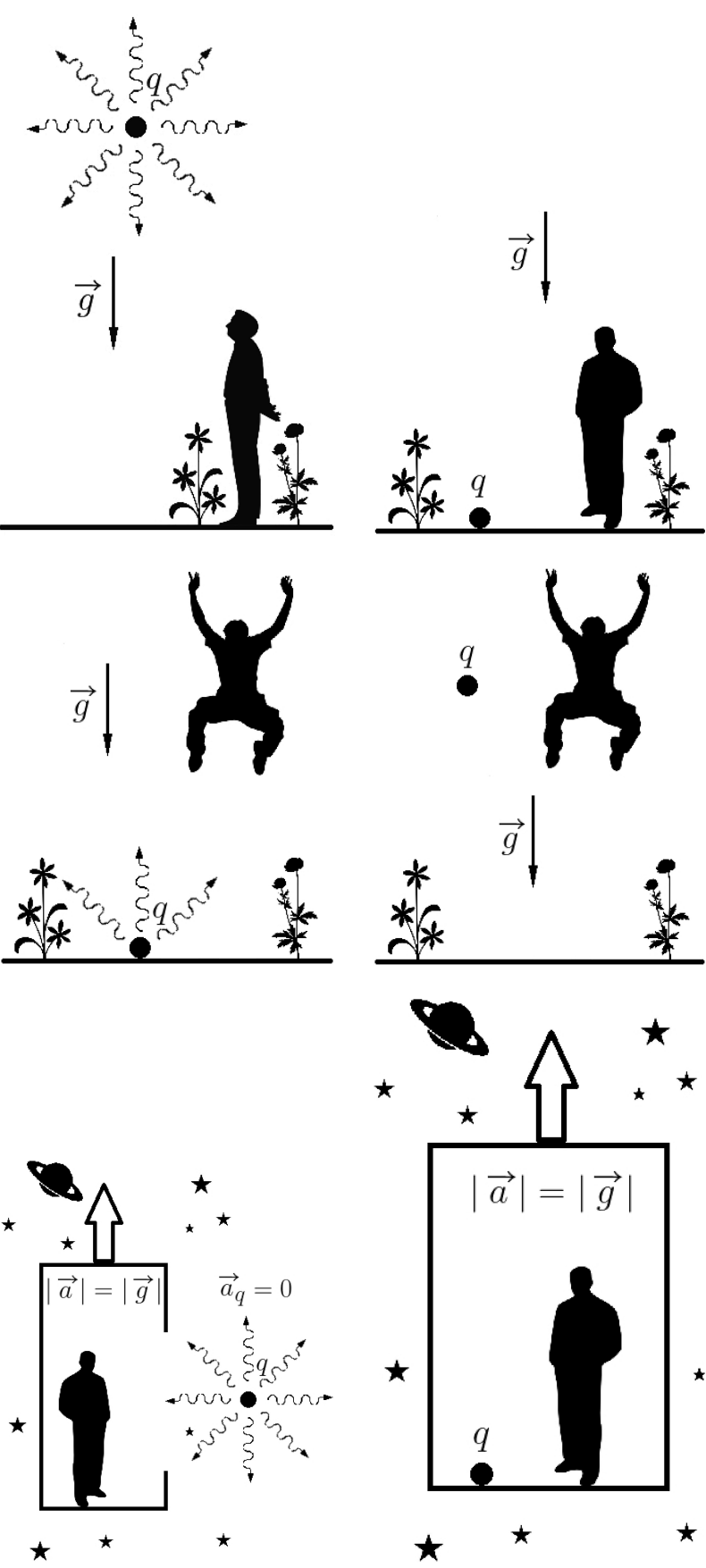

Figura 3: Representação das diversas situações envolvidas no presente artigo. A existência de radiação, indicada por linhas onduladas, é sempre em relação ao observador. Ilustrações feitas a partir de imagens encontradas em https://publicdomain vectors.org/ e https://www.hiclipart.com/

\section{Agradecimentos}

O autor agradece ao CNPq pelo apoio financeiro (processos 151967/2019-9 e 313563/2019-5). O autor agradece também ao INPE por toda a infraestrutura colocada à disposição.

\section{Referências}

[1] S. Weinberg, Gravitation and cosmology: principles and applications of the general theory of relativity (John Wiley \& Sons, New Jersey, 1972).

[2] O.A. Acevedo, E.M. de Morais and B.M. Pimentel, Rev. Bras. Ensino Fís. 41, e20180329-1 (2019).

[3] H.M. Nussenzveig, Curso de física básica (Editora Edgard BlÃ $1 / 4$ cher LTDA, São Paulo, 2013), $5^{\mathrm{a}}$ ed., v. 4.

[4] J.D. Jackson, Classical electrodynamics (John Wiley \& Sons Inc., New Jersey, 1999), $3^{\mathrm{a}}$ ed.

[5] F. Rohrlich, Ann. Phys. (N. Y.) 22, 169 (1963).

[6] F. Rohrlich, Classical charged particles (World Scientific Publishing Co. Pte. Ltd., Singapore, 2007), $3^{\mathrm{a}}$ ed.

[7] T. Fulton and F. Rohrlich, Ann. Phys. (N. Y.) 9, 499 (1960).

[8] D.G. Boulware, Ann. Phys. (N. Y.) 124, 169 (1980).ar

[9] G.E.A. Matsas, Gen. Relat. Gravit. 26, 1165 (1994).

[10] E. Eriksen and Ø. Grøn, Ann. Phys. (N. Y.) 286, 320 (2000).

[11] E. Eriksen and Ø. Grøn, Ann. Phys. (N. Y.) 286, 343 (2000).

[12] E. Eriksen and Ø. Grøn, Ann. Phys. (N. Y.) 286, 373 (2000).

[13] E. Eriksen and Ø. Grøn, Ann. Phys. (N. Y.) 297, 243 (2002).

[14] E. Eriksen and Ø. Grøn, Ann. Phys. (N. Y.) 313, 147 (2004).

[15] N.A. Lemos, Mecânica clássica (Editora Livraria da Física, São Paulo, 2004).

[16] R. d'Inverno, Introducing Einstein's relativity (Oxford University Press Inc., New York, 1995).

[17] D.J. Griffiths, Introduction to electrodynamics (Prentice Hall, New Jersey, 1999), $3^{\text {a }}$ ed.

[18] C.W. Misner, K.S. Thorne and J.A. Wheeler, Gravitation (W. H. Freeman and Company, San Francisco, 1973).

[19] W. Rindler, Relativity: special, general, and cosmological (Oxford University Press Inc., New York, 2006), $2^{\text {a }}$ ed.

[20] A. Karlhede, Gen. Relat. Gravit. 38, 1109 (2006).

[21] M. Goto, Rev. Bras. Ensino Fís. 31, 1806 (2009).

[22] N. Rosen, Ann. Phys. (N. Y.) 17, 269 (1962).

[23] C. Møller, Mat. Fys. Medd. Dan. Vid. 20, 3 (1943).

[24] R.Z. Feynman, Feynman lectures on gravitation (Addison-Wesley, Massachusetts, 1995), Lecture 9.

[25] D.R. Rowland, Eur. J. Phys. 31, 1037 (2010).

[26] Ø. Grøn, Adv. Math. Phys. 2012, ID-528631 (2012).

[27] C. de Almeida and A. Saa, Am. J. Phys. 74, 154 (2006). 